\title{
Trajetórias assistenciais de idosos em uma região de saúde do Distrito Federal, Brasil
}

\author{
Healthcare pathways of elderly in one health region in the Federal \\ District, Brazil
}

Ruth da Conceição Costa e Silva Sacco', Marcella Guimarães Assis², Raíssa Gomes Magalhães', Sílvia Maria Ferreira Guimarães', Patrícia Maria Fonseca Escalda1

DOI: 10.1590/0103-1104202012618

RESUMO O objetivo foi identificar as trajetórias assistenciais de idosos em uma região de saúde do Distrito Federal e suas percepções acerca desses percursos. Pesquisa qualitativa que analisou, com base na metodologia de caso traçador, 14 percursos assistenciais de idosos. Foram realizadas entrevistas com idosos e cuidadores, processadas por análise de conteúdo; e consulta a prontuários. Mapas das trajetórias descritas foram elaborados por geoprocessamento. Identificou-se que o percurso real feito pelos idosos difere do previsto na legislação, e que as fragilidades apontadas estiveram relacionadas à difícil acessibilidade, ao deficit de profissionais, à falta de medicamentos e ao tempo de espera para atendimento na atenção especializada. As potencialidades relatadas pelos idosos envolveram humanização no atendimento, tais como o bom acolhimento por parte da equipe, a construção de vínculos e escuta qualificada, e sensação de bem-estar após as consultas. Apesar de o modelo de atenção ainda ser fragmentado e estar distante da situação ideal, os usuários se sentem satisfeitos com os cuidados recebidos, evidenciando aspectos positivos no processo do cuidar em saúde. Entretanto, a organização de processos de trabalho e a articulação entre os níveis de atenção à saúde precisam ser revistas e adequadas às especificidades desse ciclo de vida.

PALAVRAS-CHAVE Aceitação pelo paciente de cuidados de saúde. Serviços de saúde para idosos. Acesso aos serviços de saúde. Assistência à saúde. Saúde do idoso.

1 Universidade de Brasília (UnB) - Brasília (DF),

Brasil.

ruth.sacco1@gmail.com

2 Universidade Federal de Minas Gerais (UFMG)

- Belo Horizonte (MG), Brasil.

\begin{abstract}
The aim was to identify the healthcare pathways of the elderly in one health region in the Federal District and their perceptions about these pathways. A qualitative study analyzed 14 healthcare pathways of elderly people, using tracer methodology. Interviews were conducted with the elderly and caregivers, processed by content analysis; and medical records were reviewed. Maps of the paths described were prepared by geoprocessing. It was found that the actual healthcare pathways taken by the elderly differ from what law would predict, and that the weaknesses detected were related to poor accessibility conditions, lack of professionals, lack of medicines, and the long waiting time for specialized health care. The expectations reported by the elderly involved humanization of care, emerging elements such as being well received by the personnel, the building of bonds, qualified listening; and a feeling of well-being after the medical consultation. Although the care model is still fragmented and far from ideal, users feel satisfied with the care provision and identify positive aspects in the health care process. However, the organization of work processes and the articulation between levels of health care need to be reviewed and adapted to the specificities of this life phase.
\end{abstract}

KEYWORDS Patient acceptance of health care. Health services for the aged. Health services accessibility. Delivery of health care. Health of the elderly. 


\section{Introdução}

O envelhecimento da população é reflexo da transição demográfica, resultante do declínio nas taxas de fecundidade e de mortalidade ${ }^{\mathbf{1}}$, e representa um desafio, haja vista a necessidade de desenvolvimento de políticas e cuidados de saúde adequados para idosos ${ }^{2}$. No Brasil, o número de idosos ( $\geq 60$ anos de idade) estimado para 2020 é de 32 milhões, tendo um aumento de mais de $200 \%$ em relação a 2002 , ano em que chegava a 14 milhões ${ }^{2}$. No Distrito Federal (DF), seguindo a tendência nacional, a população idosa aumentou de 7,7\%, em 2010, para $10,5 \%$, em $2018^{3}$.

Apesar de a redução de doenças infectocontagiosas ter contribuído para o aumento da expectativa de vida ${ }^{4}$, elevou-se a prevalência de doenças crônico-degenerativas que afetam, sobretudo, idosos 5 . Este cenário aponta a necessidade de adoção de estratégias de promoção do envelhecimento saudável ${ }^{6}$, definido pela Organização Mundial da Saúde como processo de desenvolvimento e de manutenção da capacidade funcional, que permite o bem-estar em idade avançada?.

Diante disso, o Sistema Único de Saúde (SUS) tem se adequado para garantir ações e serviços qualificados e resolutivos, organizando-os em redes. A discussão sobre Redes Integradas de Serviços de Saúde (Riss) emergiu com a Resolução CD49.R22, de 02 de outubro de 2009, da Organização Pan-Americana da Saúde, e propõe a superação de sistemas fragmentados e hierarquizados ${ }^{8}$.

As Riss podem ser definidas como

Una red de organizaciones que presta, o hace los arreglos para prestar, servicios de salud equitativos e integrales a una población definida, y que está dispuesta a rendir cuentas por sus resultados clínicos y económicos y por el estado de salud de la población a la que sirve8(11).

Elas objetivam desenvolver a Atenção Primária à Saúde (APS) enquanto base do sistema, e a prestação de serviços adequados às expectativas dos usuários, com acessibilidade, equidade, eficiência e qualidade técnica ${ }^{8}$. No Brasil, são chamadas de Redes de Atenção à Saúde (RAS) e são coordenadas pela APS, primeiro contato preferencial dos usuários e centro de comunicação com toda a RAS ${ }^{1,9}$. Assim, a APS busca evitar a concentração de serviços e investimentos nos níveis secundário e terciário de atenção à saúde10,11.

A Estratégia Saúde da Família (ESF), enquanto modelo de atenção sugerido para a APS, enfatiza a integralidade das ações e focaliza o indivíduo integrado à família ${ }^{12}$, promove acolhimento e escuta qualificada para a resolução de demandas ${ }^{\mathbf{1 3}}$ e é responsável por orientar o percurso dos usuários dentro da $\mathrm{RAS}^{14}$. A análise deste percurso (trajetória assistencial) possibilita identificar fragilidades e potencialidades na $\operatorname{RAS}^{15}$ e evidencia a percepção dos usuários sobre a qualidade do cuidado ${ }^{16}$.

Considerando o envelhecimento da população e as especificidades requeridas pelo novo perfil epidemiológico, assim como os pressupostos das RAS e da APS, o presente estudo identificou trajetórias assistenciais de idosos em sua busca por assistência, em uma região de saúde do DF.

\section{Material e métodos}

Estudo qualitativo que identificou trajetórias assistenciais de idosos por meio da metodologia do caso traçador ${ }^{\mathbf{1 7}}$. Foram realizadas entrevistas semiestruturadas com idosos e cuidadores, e consultas a prontuários, que serviram de marcadores para a análise dos processos de cuidado e para o reconhecimento de como estes se concretizam no cotidiano em saúde ${ }^{18}$.

Foi conduzido na Região de Saúde Oeste (RSO) do DF, que inclui duas Regiões Administrativas (RA): Ceilândia e Brazlândia. A primeira, com 432.927 habitantes (7,24\% são idosos), é a mais populosa do DF; e a segunda possui 53.534 habitantes (7,01\% são idosos) ${ }^{19}$. A RSO foi escolhida porque nela há elevado 
percentual de idosos vulneráveis, com maior risco de declínio funcional e de morte ${ }^{20}$. Foram envolvidas cinco equipes de Saúde da Família (eSF), de quatro Unidades Básicas de Saúde (UBS), sendo três da Ceilândia e uma de Brazlândia, selecionadas com base na estrutura etária das respectivas áreas de abrangência.

Seguindo essa técnica, cada equipe foi solicitada a apontar 3 casos significativos do perfil do serviço: 1 típico, 1 de sucesso e 1 de tensão ${ }^{\mathbf{1 8}}$, totalizando 15 casos traçadores. Os critérios de inclusão foram: ser residente na RSO e ser $\geq 18$ anos (se entrevista conduzida com o cuidador, em situações de limitação cognitiva do idoso). Os de exclusão foram: indivíduos não localizados após três tentativas de contato em horários e por meios de comunicação distintos.

O roteiro da entrevista foi construído após visitas exploratórias ${ }^{21}$, e continha nove perguntas relacionadas ao motivo da ida à UBS; à percepção sobre o cuidado; à trajetória percorrida em busca de assistência; e às condições atuais de saúde do idoso e a relação desta com a APS. As informações foram gravadas (gravador Sony ICD-PX240), transcritas e interpretadas. Fez-se análise semântica do conteúdo, partindo-se das categorias de análise que emergiram do material ${ }^{22}$.

As entrevistas subsidiaram a elaboração de mapas por geoprocessamento de três trajetórias assistenciais, utilizando-se o Sistema de Referência Geocêntrico para as Américas (Sirgas) ${ }^{23}$, para os quais se fez infográfico.

Esta pesquisa foi aprovada em Comitê de Ética, sob os pareceres 2.202.975 (Universidade de Brasília) e 2.269.757 (Secretaria de Saúde-SES/ DF). Todos os participantes assinaram o Termo de Consentimento Livre Esclarecido (TCLE).

\section{Resultados e discussão}

Neste estudo, dos 15 casos indicados pelas UBS, 1 foi excluído pela inexistência de retorno às tentativas de contato. Assim, identificaram-se trajetórias assistenciais de 14 idosos e suas percepções acerca da assistência à saúde no DF. Os resultados mostraram que a trajetória prevista na legislação, com base nos normativos e protocolos da SES/DF, divergiu da que efetivamente foi feita pelos idosos. Entretanto, apesar das fragilidades por eles apontadas para o funcionamento da RAS, todos eles consideraram-se satisfeitos com o atendimento recebido e com suas atuais situações de saúde.

A maioria dos idosos participantes era da faixa etária de 70 a 79 anos (42,9\%), do sexo feminino $(57,1 \%)$ e composta por viúvos (85,7\%). A tabela 1 apresenta características sociodemográficas destes idosos.

Tabela 1. Caracterização dos idosos (casos traçadores), das Unidades Básicas de Saúde (UBS) selecionadas, Região de Saúde Oeste, Distrito Federal, 2019

\begin{tabular}{lcr}
\hline Variáveis & N & \% \\
\hline Idade & 3 & 21,4 \\
$60-69$ anos & 6 & 42,9 \\
$70-79$ anos & 5 & 35,7 \\
$80-89$ anos & & 42,9 \\
Sexo & 6 & 57,1 \\
Masculino & 8 & 14,3 \\
Feminino & & 85,7 \\
Estado civil & 2 & 12 \\
Casado & 12 & \\
Viúvo & & \\
\hline
\end{tabular}


Tabela 1. (cont.)

\begin{tabular}{lrr}
\hline Variáveis & N & \% \\
\hline Arranjo familiar & 12 & 85,7 \\
Mora acompanhado & 2 & 14,3 \\
Mora sozinho & $\mathbf{1 4}$ & $\mathbf{1 0 0}$ \\
\hline Total de Idosos & & \\
\hline
\end{tabular}

Fonte: Elaboração própria.

Apesar de a maioria deles ser composta por viúvos $(85,7 \%)$ - o que pode trazer implicações, pois a viuvez relaciona-se a prognósticos mais desfavoráveis em saúde e à mortalidade precoce $^{24}$-, 92,9\% dos idosos moravam com algum familiar, inferindo-se que são indivíduos que têm rede de suporte social, aspecto importante na adesão ao tratamento ${ }^{25}$.
Quanto à situação de cadastramento na ESF, verificou-se que, maiormente, ele foi realizado por demanda espontânea em situações de busca para tratamento de doença, enfatizando que há uma deficiência no cadastramento feito pelas UBS, ocasionado por falha no processo de organização do trabalho ou deficit de profissionais ${ }^{26}$ (figura 1).

Figura 1. Origem e tempo de cadastramento dos idosos (casos traçadores) na Estratégia Saúde da Família (ESF), das Unidades Básicas de Saúde (UBS) selecionadas, Região de Saúde Oeste, Distrito Federal, 2019
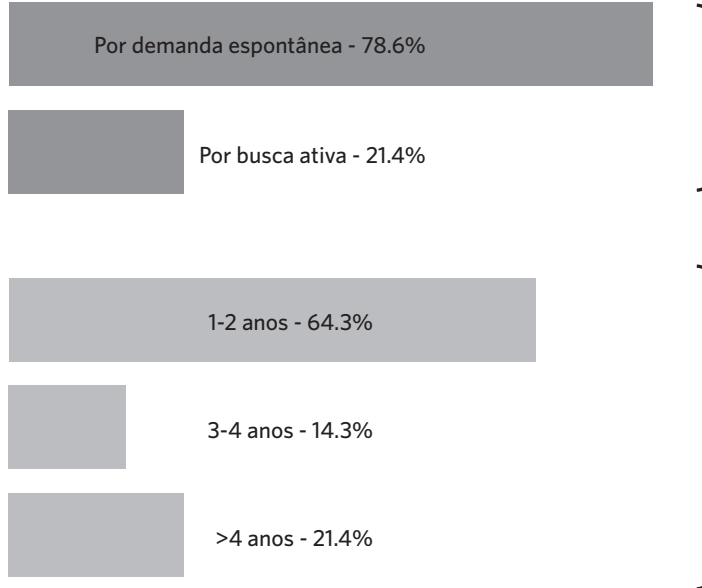

\section{$1-2$ anos $-64.3 \%$}

3-4 anos $-14.3 \%$

$>4$ anos $-21.4 \%$

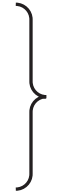

Origem do cadastro na Estratégia Saúde da Família

Tempo de cadastramento na Estratégia Saúde da Família

Fonte: Elaboração própria.

No DF, havia experiências isoladas da ESF realizadas por algumas equipes, entretanto, apenas em 2017, elegeu-se esse modelo de atenção enquanto estruturante da APS, utilizando-se de força de trabalho já existente nas $\mathrm{UBS}^{27}$. Assim, provavelmente este panorama justifique o pouco tempo de cadastramento dos idosos na ESF, tendo, a maioria, dois anos.

\section{As trajetórias assistenciais dos idosos}

Trajetórias assistenciais são percursos escolhidos pelos indivíduos, que ocorrem dentro da rede de saúde, e que podem ou não conseguir resolutividade e tratamento ${ }^{15}$. Aqui, faz-se contraponto à definição de itinerário 
terapêutico, que envolve a busca de cuidados em saúde pelos indivíduos, que, na busca de soluções para seus problemas, excedem os serviços de saúde, e sua análise considera aspectos socioculturais, que influenciam os percursos individuais ${ }^{\mathbf{2 8}, 29}$. Neste estudo, optou-se por traçar o percurso enquanto trajetórias assistenciais, pela necessidade de identificação de fluxos entre os níveis de atenção.

Os idosos participantes possuíam comorbidades, tais como: diabetes mellitus, hipertensão arterial sistêmica, perda de visão limitadora de realização das Atividades de Vida Diária (AVD), demência, dores articulares, fibrose pulmonar, neoplasias e doenças infecciosas de notificação compulsória, o que provavelmente os fazia percorrer serviços de saúde, públicos e/ou privados. Os mapas por geoprocessamento foram elaborados para apenas 3 dos 14 casos analisados, uma vez que a falta de registros em prontuário, o viés de memória e a ausência de documentos por parte dos idosos e/ou seus familiares, prejudicou a identificação e a confirmação dos endereços dos serviços assistenciais por onde haviam transitado. As trajetórias reais foram sobrepostas às previstas, conforme sugerido nos normativos que regem as RAS no DF, e o resultado é apresentado na figura 2.

Figura 2. Trajetórias assistenciais de idosos na Região de Saúde Oeste, Distrito Federal, 2019

\section{2.a UBS D, 12, queixa 'zumbido no ouvido'}

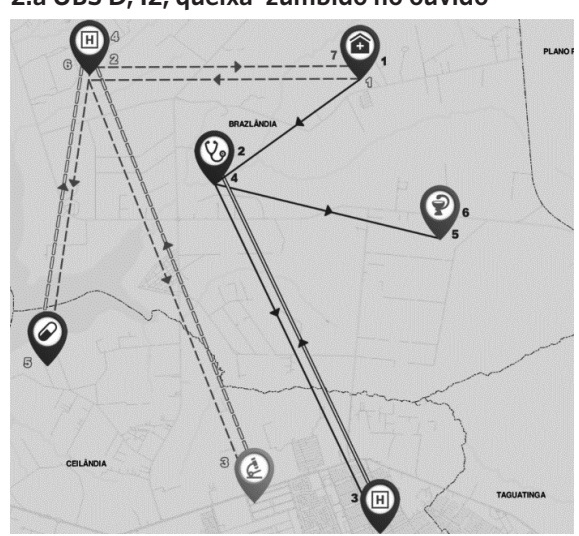

2.b UBS D, I1, queixa 'tosse persistente'.

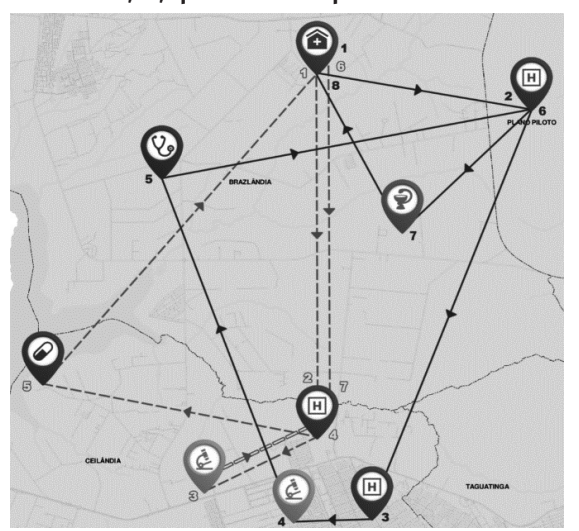

\begin{tabular}{ll}
\hline Real & Previsto \\
\hline 1 Unidade Básica de Saúde № 5 Brazlândia & 1 Unidade Básica de Saúde № 5 Brazlândia \\
2 Otorrinolaringologista Particular & 2 Hospital Regional de Brazlândia \\
3 Hospital das Clínicas & 3 Laboratório de Ceilândia \\
4 Otorrinolaringologista Particular (Retorno) & 4 Hospital Regional de Brazlândia \\
5 Drogaria Particular (Consulta) & 5 Farmácia Ambulatorial Especializada da \\
& Ceilândia \\
6 Drogaria Particular (Compra) & 6 Hospital Regional de Brazlândia \\
& 7 Unidade Básica de Saúde № 5 Brazlândia \\
\hline
\end{tabular}

\begin{tabular}{ll}
\hline Real & Previsto \\
\hline 1 Unidade Básica de Saúde № 5 Brazlândia & 1 Unidade Básica de Saúde № 5 Brazlândia \\
2 Hospital de Base & 2 Hospital Regional de Ceilândia \\
3 Hospital das Clínicas & 3 Laboratório de Ceilândia \\
4 Laboratório Particular & 4 Hospital Regional de Ceilândia \\
5 Exames Cardiológicos Particular & 5 Farmácia Ambulatorial Especializada da \\
& Ceilândia \\
6 Hospital de Base & 6 Unidade Básica de Saúde № 5 Brazlândia \\
7 Drogaria Particular (Compra) & 7 Hospital Regional de Ceilândia \\
\hline
\end{tabular}


Figura 2. (cont.)

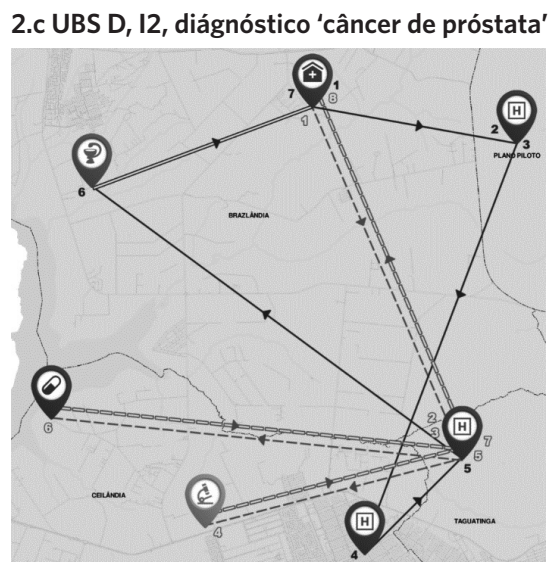

Equipamentos de Saúde

(10) Hospitais Gerais

() Unidades Básicas de Saúde

(2) Consultório

(e) Laboratório

(2) Farmácia Ambulatorial

(Q) Farmácia/Drogaria

\begin{tabular}{ll}
\hline Real & Previsto \\
\hline 1 Unidade Básica de Saúde № 5 Brazlândia & 1 Unidade Básica de Saúde № 5 Brazlândia \\
2 Hospital de Base (Consulta) & 2 Hospital Regional de Taguatinga (Con- \\
& sulta) \\
3 Hospital de Base (Exames) & 3 Hospital Regional de Taguatinga (Exames) \\
4 Hospital Anchieta (Radioterapia) & 4 Laboratório de Ceilândia (Exames) \\
5 Hospital Regional de Taguatinga (Acom- & 5 Hospital Regional de Taguatinga (Radio- \\
panhamento) & terapia) \\
6 Drogaria Particular (Compra) & 6 Farmácia Ambulatorial Especializada da \\
& Ceilândia \\
7 Unidade Básica de Saúde № 5 Brazlândia & 7 Hospital Regional de Taguatinga (Retorno) \\
& 8 Unidade Básica de Saúde № 5 Brazlândia \\
& (Retorno) \\
\hline
\end{tabular}

Deslocamento

$-\rightarrow-$ Fluxo previsto

$\Rightarrow$ Retorno previsto

$\rightarrow$ Fluxo realizado

$\Longrightarrow$ Retorno realizado

Fonte: Elaboração própria.

Nota: Consultório, laboratório e farmácia/drogaria correspondem a equipamentos de saúde privados.

A figura 2.a representa percurso de idosa de 78 anos, cuja queixa era de zumbido no ouvido. Ela buscou a UBS de seu território, onde foi solicitado exame de imagem. Para realizá-lo, devido à demora no atendimento por especialista na RAS do SUS, ela buscou clínica privada de otorrinolaringologia. As figuras 2.b e 2.c referem-se a dois caminhos distintos de um mesmo idoso de 89 anos, que buscou UBS em virtude de tosse persistente (figura 2.b), tendo sido encaminhado à Atenção Especializada (AE) para a realização de exames de imagem por suspeita de tuberculose. Além disso, anos atrás, ele havia sido diagnosticado com câncer de próstata e fez seu primeiro acesso por meio da UBS, como ilustra a figura 2.c.

Nas três trajetórias apresentadas, percebe-se que a UBS da área de abrangência foi o primeiro acesso para os casos, e fez o acolhimento e o encaminhando à AE. Porém, a partir disso, o fluxo não ocorreu conforme previsto, pois, em dois dos três casos (figuras 2.b e 2.c), os idosos acessaram a rede privada, tanto para consulta com especialista quanto para realização de exames complementares, para evitar filas de espera no SUS. Este achado corrobora o estudo de Raupp et al. ${ }^{29}$, que aponta esta fragilidade como presente em todo o País. No DF, somente em 2018 ocorreu a implantação do Complexo Regulador em Saúde (CRDF), tendo a APS como ordenadora do acesso, o que poderia explicar a dificuldade de os idosos realizarem consultas e exames especializados ${ }^{30}$.

Comparando a trajetória real com a prevista, observou-se que nenhum dos casos seguiu a trajetória esperada, sendo indicativo da falta de integração entre os pontos de atenção e de falhas nas tecnologias disponíveis no sistema 
de saúde, gerando fragmentação do cuidado e percurso mais longo e dispendioso para os idosos ${ }^{12,31}$, além de torná-los mais propensos a desenvolverem outras comorbidades ${ }^{32,33}$. O acompanhamento longitudinal feito dentro do serviço de saúde, especialmente pela APS, confirma a eficiência da UBS e a satisfação do usuário com o serviço. De modo contrário, estes percursos assistenciais evidenciam fragilidades no funcionamento dos fluxos de referência e contrarreferência, comprometendo o acesso à $\mathrm{AE}^{\mathbf{3 4}}$.

Os resultados mostraram que, apesar de a trajetória dos idosos ter iniciado na rede pública, houve etapas na rede privada, independentemente da complexidade dos casos, pois encaminhamentos feitos para a AE também tiveram fluxos desviados para serviços custeados pelos próprios usuários (figura 2.a). Destacou-se que, na situação de maior complexidade (tratamento de câncer de próstata, figura 2.c), em que a trajetória real esteve fora da região de saúde de residência do idoso, os equipamentos de saúde utilizados foram maiormente da RAS do SUS, ou seja, a própria rede indicou caminhos assistenciais alternativos para tratamento oportuno e gratuito da neoplasia, embora em percurso mais longo e fora da RSO.

Spedo et al. ${ }^{35}$, em estudo semelhante realizado em São Paulo (SP), afirmaram que o maior gargalo se encontrava em serviços de média complexidade, especialmente por falhas gestoras relacionadas à falta de priorização política, a despeito de existirem mecanismos, tais como o complexo regulador e a informatização das suas ações. Esses autores apontaram que deve haver uma lógica de retroalimentação entre APS e média complexidade, pois para que a APS seja resolutiva é preciso que o acesso à $\mathrm{AE}$ exista e, em contrapartida, se a APS possuir baixa resolutividade, os encaminhamentos à $\mathrm{AE}$ irão aumentar.

No DF, as trajetórias analisadas não ocorreram como previstas, demonstrando fluxo real fragmentado e decorrente de fragilidades nos sistemas logísticos que envolvem o CRDF, situação que gera comprometimento do acesso aos níveis especializados e da integralidade da atenção, tornando o percurso do idoso mais longo e financeiramente dispendioso ${ }^{33}$.

\section{Percepção dos idosos acerca dos cuidados à saúde do idoso na APS}

Para que a lacuna entre a teoria e a realidade do SUS não inviabilize a concretização da RAS, além da análise dos caminhos percorridos em busca de cuidados em saúde, deve-se considerar a perspectiva dos usuários e sua satisfação com a atenção recebida, enquanto estratégias para a avaliação do cuidado ofertado ${ }^{36}$.

A análise de conteúdo das entrevistas com os idosos permitiu a identificação de quatro categorias de análise distintas, todas correlacionadas a princípios e/ou diretrizes do SUS e à APS (quadro 1).

Quadro 1. Categorias de análise identificadas e características observadas nestas categorias

\begin{tabular}{ll}
\hline Categorias de Análise & Eixos Temáticos Envolvidos \\
\hline Humanização no atendimento & Acolhimento, vínculo, escuta qualificada e bem-estar \\
Organização de processos de trabalho & Acesso à Unidade Básica de Saúde \\
Recursos organizacionais & Recursos humanos (deficit e suficiência) \\
& Recursos materiais e infraestrutura (deficit e suficiência) \\
Atendimento na atenção especializada & Lista de espera e comunicação entre os níveis de atenção \\
\hline
\end{tabular}

Fonte: Elaboração própria. 
As percepções dos usuários relacionaram-se tanto a potencialidades quanto a fragilidades na linha de cuidado ao idoso, no DF, e as principais delas são apresentadas na figura 3.

Figura 3. Fragilidades e potencialidades na linha de cuidado ao idoso, Região de Saúde Oeste, Distrito Federal, 2019

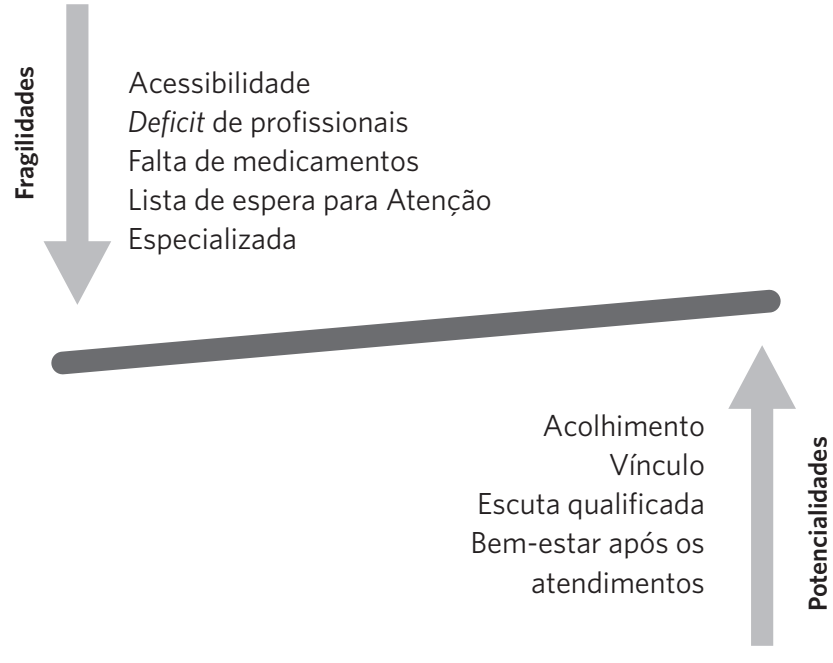

Fonte: Elaboração própria.

\section{HUMANIZAC̣̃̃O NO ATENDIMENTO}

Nas práticas centradas no usuário, faz-se necessário desenvolver habilidades para adequado acolhimento e estabelecimento de vínculo entre equipe, profissionais e usuários ${ }^{27}$. Assim, o acolhimento deve ser visto como dispositivo potente e diretriz operacional para o sistema de saúde, sendo útil para a identificação das demandas, a construção de vínculo profissional-usuário e o cuidado integral, atendendo aos princípios da $\mathrm{APS}^{37-39}$.

Neste estudo, o acolhimento e a escuta qualificada foram apontados como potencialidades, e os relatos estiveram associados à qualidade e à sensação de bem-estar após os atendimentos, como apresentado abaixo:

Eles conversam com a gente, explicam tudo com a maior paciência, com o maior carinho, entendeu? Isso, pra mim, é muito importante, que eu sou uma pessoa assim [...] eu fico assim, olha, pode ser... Me agradar aquela coisinha, aquela minha amizade, amizade pura. (UBS C, 11).

Eu não sei, elas falavam assim: 'Dona Fulana, qualquer coisa me procure'. Elas me abraçam. Essas meninas todas me abraçam assim, como uma mãe assim [referindo-se às profissionais da enfermagem]. Continuo indo lá. É sempre as mesmas pessoas que me atendem. Eu sempre fui bem atendida ali. Até na hora que eu vou lá só pra pegar receita, eu sou hiper bem atendida. (UBS A, 14).

Notou-se que a satisfação dos idosos estava diretamente relacionada ao acolhimento e ao vínculo, demonstrando sua importância para a continuidade do cuidado e destacando-os como potencialidades da APS. Resultado semelhante foi identificado por Uchoa et al. $^{40} \mathrm{e}$ por Vello et al.' ${ }^{1}$ que destacaram o acolhimento e o vínculo como as dimensões do cuidado mais satisfatórias para os usuários. 
O estabelecimento de vínculos pressupõe a construção de relações de afetividade e confiança ${ }^{36}$, e os laços entre os envolvidos se fortalecem quando o convívio se torna frequente, contribuindo para a eficácia do tratamento, e evitando consultas e internações desnecessárias, pois usuários aderem melhor aos tratamentos propostos ${ }^{41}$. Estas tecnologias relacionais são especialmente importantes para os idosos que, em sua maioria, apresentam doenças crônicas não transmissíveis, dependem de tratamento medicamentoso e necessitam de cuidados continuados em saúde.

\section{ORGANIZAC̣ÃO DE PROCESSOS DE TRABALHO}

Os idosos relataram sua dificuldade de acesso à UBS, o que demonstrou a pouca organização dos processos de trabalho. Entre os atributos da APS está a acessibilidade, definida com a oferta de serviços pela RAS, pois, para o usuário adentrar os serviços oferecidos e usufruí-los, ele deve poder acessá-los ${ }^{38}$. Acesso e acessibilidade têm sentidos próximos, haja vista que se relacionam à capacidade, não só de produzir e ofertar serviços, mas também de torná-los responsivos às necessidades de saúde da população. Aqui, utilizou-se o conceito de acessibilidade que inclui as dimensões geográfica (distância, tempo e custo de locomoção) e sócio-organizacional (capacidade de acolher às demandas, adequada e oportunamente) $\mathbf{4 2}^{\mathbf{2}}$.

Em relação à acessibilidade, os idosos relataram fragilidades importantes da APS:

[...] que pode mudar um pouco... O que eu acho assim, na marcação de consulta. Porque a gente vai muito cedo pra marcar uma consulta. Tem vez que eu saio daqui 5h30min da manhã pra 'tá lá na fila. Mesmo idoso. A gente vai pra filinha lá de lado de fora. Um frio! Mesmo com a ESF, ainda tem que pegar fila. (UBS C, 11).

Aqui é ruim pr'a gente ir ao Posto. São as condições, a gente não tem carro... Pra você ver: eu ainda vou ao Posto hoje. Agora, eu 'tô aguentando andar. E quando eu não aguentar mais? (UBS D, 12).
Segundo Franco et al.43, a reestruturação da APS deu-se com a implementação da ESF para garantir a organização no processo de trabalho e atendimento a todos os usuários que procurem a unidade, sem a necessidade de fichas e filas de madrugada. No entanto, a fala do usuário 'UBS C, Il' demonstra que, mesmo após 25 anos da implementação da ESF ter ocorrido no Brasil, e considerando que se passaram 2 anos da adoção da ESF no DF, ainda existem UBS que trabalham no modelo tradicional, submetendo os usuários a longas filas de espera para serem atendidos.

A distância do local de atendimento relatada pelo usuário 'UBS D, I2' demonstra realidade adversa da recomendação feita por Silva et al. ${ }^{44}$, que afirmam que a proximidade do local de atendimento é um dos aspectos fundamentais na APS, a qual deve oferecer serviços de forma planejada e considerando a localização geográfica da UBS, que deve ficar estrategicamente perto das moradias da população para aprimorar a acessibilidade, principalmente se há idosos.

A realização de visitas domiciliares pelos profissionais de saúde, por oportunizar contato direto com usuários, proporciona satisfação aos idosos. Em sua pesquisa, Conill ${ }^{45}$ identificou visitas como bem avaliadas pelos entrevistados. No presente estudo, idosos demonstraram maior sensação de bem-estar e satisfação quando as visitas domiciliares faziam parte de seus planos de cuidados, e reivindicaram maior frequência desse tipo de atividade.

Eu adoro as meninas. Um dia, elas chegaram aqui tudinho na minha casa, minha pressão subiu de tanta alegria. A senhora acredita? Porque elas vieram aqui me visitar, me dar atenção, saber da minha saúde. Vieram cuidar de mim. (UBS C, 11).

Se viessem mais, pelo menos, uma vez ao mês [referindo-se à frequência de visitas domiciliares]... É até bom... Eu digo, sei que não é pra isso que vocês fazem, mas, ao menos, um pouco de conversa com a gente. Aí, me sentiria feliz. 
Ele falava: 'Terça-feira eu volto'. Aí, você ficava naquela ansiedade, e ele não vinha [referindo-se ao enfermeiro, que agendava a visita domiciliar, mas não comparecia]. (UBS C, 12).

Também foram percebidos esforços para que o atendimento aos idosos fosse priorizado, talvez para o cumprimento do Estatuto do Idoso (EI), que lhes confere essa garantia em órgãos públicos e privados ${ }^{46}$. Os registros de alguns usuários evidenciaram situações nas quais o acesso esteve facilitado:

Davam prioridade devido à idade dele [familiar referindo-se ao usuário idoso], a situação de ser uma pessoa idosa que não tem familiar. Então, muitas vezes, eu vi que as funcionárias já sabiam do caso, já acompanhavam, até mesmo pela forma como ele estava, debilitado, demente, já. O acoIhimento foi diferenciado. (UBS A, I1).

Toda vez que eu vou, não volto sem atendimento. Tanto faz, estar marcado como não. Alguém dá um jeito e me consulta. O povo aqui já me conhece e sempre dá um jeito de me atender. Os idosos entram primeiro. (UBS C, I1).

Em estudo com equipes multiprofissionais em São Paulo verificou mudanças positiva no comportamento dos envolvidos no atendimento a idosos após a publicação do EI e constatou, sobretudo, maior respeito no atendimento ${ }^{47}$. Em Salgueiro, interior de Pernambuco, idosos entrevistados acerca da satisfação com o atendimento na APS, informaram que suas expectativas eram atendidas sempre que buscavam o serviço (82,3\%). Entretanto, quanto à prioridade, 38,5\% afirmaram não haver diferenciação, enfatizando que, em algumas UBS, idosos eram atendidos com os mesmos critérios de usuários de outras idades ${ }^{48}$.

\section{RECURSOS ORGANIZACIONAIS DA APS}

A falta de medicamentos e de materiais médicos para usuários demonstra a fragilidade do princípio da gratuidade do SUS, o qual não deve gerar custos financeiros para idosos, e deve garantir assistência farmacêutica ${ }^{49}$. Idosos são usuários frequentes de serviços de saúde e, em sua maioria, necessitam de medicamentos ao longo da vida ${ }^{50}$. Assim, essa indisponibilidade afetou a vida dos usuários idosos.

É porque eu tomo muito remédio, né? Tem vez que a gente não acha, que a gente precisa comprar do bolso. Eu tomo ele direto. Quando não tem, tenho que tirar do bolso. Às vezes, pego dinheiro emprestado pra comprar o remédio. (UBS D, 12).

Aí, sempre que tem lá, eu pego. O difícil é a fita da glicemia. O governo não está mandando. Eu ainda tenho uma caixinha aí. Trouxe de lá mesmo, mas agora não está tendo. (UBS D, 12).

Primeira vez que veio pra cá, prometeu que ia arranjar um cilindro desse pra mim, desse de transporte [idosa portadora de fibrose cística, referindo-se a cilindro de oxigênio portátil que o enfermeiro da eSF lhe prometeu em visita domiciliar]. Não deu resposta. Ficou sem saber. Eu sei que é uma correria danada, lá é fervendo, né? (UBS B, 12).

Somando-se a isso, a infraestrutura deficitária também foi apontada por um usuário enquanto dificultadora da resolutividade das ações assistenciais.

Está tudo jogado às traças [referindo-se à gestão do Governo local], porque você vai no hospital aí, isso é uma pouca vergonha. Você vai nesse Posto de Saúde aí, meu Deus do céu, um descaso! (UBS A, 13).

Para Paim ${ }^{51}$, entre os aspectos negativos da consolidação do SUS, citam-se políticas de medicamentos e de assistência farmacêutica, e infraestrutura insipiente. Para ele, isto pode ser resultante de investimentos limitados no setor saúde pública no Brasil, que se refletem em dificuldades na manutenção de serviços e na ampliação da infraestrutura.

Além da falta de medicamentos e insumos, os idosos perceberam carência no quadro de 
profissionais de saúde. A eSF deve ser composta por, no mínimo, 1 médico de família ou generalista, 1 enfermeiro, 1 auxiliar de enfermagem e 1 Agente Comunitário de Saúde (ACS) para cada 750 pessoas 9 . Porém, muitas vezes, este número de profissionais não é suficiente devido à grande área para cobertura e à alta demanda. Somando-se à desestruturação dos processos de trabalho, isto pode implicar na perda da qualidade dos serviços ofertados, além de ocasionar sobrecarga profissional, com vê-se a seguir:

O doutor está muito sozinho, tem muito serviço pra ele. Devia ter, pelo menos, uns três médicos mesmo, né? Porque é muito serviço pra ele, gente! O pobre vai pra um canto, vai pro outro. Não tem quem aguente o tanto de paciente que ele atende. (UBS $B, 11$ ).

Só o que eu te falei daquele negócio, né? [sobre os profissionais de saúde que faltam muito], que tem mês que eles não vêm. Não sei, é só porque eles pegam atestado, mas é porque a equipe também está reduzida. (UBS A, I3).

A fala do idoso 'UBS B, Il' ilustra consciência sanitária, na medida em que o usuário compreende que há deficit de profissionais e reconhece que estes estão sobrecarregados. O reconhecimento da população e sua participação na organização, na gestão e no controle das ações e dos serviços de saúde têm grande contribuição para melhorias de políticas públicas, pela importância da divisão de responsabilidades entre usuários e SUS ${ }^{52}$. A pressão exercida sobre a equipe evidencia a desorganização da oferta do cuidado e aponta a necessidade de estratégias e competências para lidar com o envelhecimento.

Considerando a reforma da APS (Converte APS), através da qual tornou-se obrigatória a ESF em todas as UBS do DF, destacou-se a pouca adesão por parte dos médicos, o que poderia justificar a situação acima relatada. Os médicos que optaram por não permanecer na APS foram realocados em outros níveis de atenção à saúde, sucedendo-se ações para a manutenção do atendimento mínimo à população, tais como a criação do cargo de enfermeiro especialista em saúde da família e comunidade e a realização de concurso para a contratação desses enfermeiros e de médicos especialistas nessa mesma área. Entretanto, gargalo ainda maior ocorre no deficit de ACS, cuja necessidade é de 3 mil profissionais e, atualmente, há apenas o mínimo para a habilitação de equipes, totalizando mil $\mathrm{ACS}^{27}$.

\section{ATENDIMENTO NA AE}

A operacionalização da AE no SUS prescinde da organização em RAS, a qual é responsável pelo cumprimento do princípio da integralidade do cuidado, evitando a fragmentação dos serviços prestados nos diferentes níveis de complexidade ${ }^{30,53}$.

Quando questionados se já haviam buscado atendimento em outros serviços, e como se efetivaram as ações de saúde após a consulta na UBS, os idosos relataram suas experiências negativas com encaminhamentos para os níveis especializados, de média e alta complexidade.

Aí, ela pediu pra eu fazer um exame, mas nem médico nem ninguém chama. Os hospitais não chamam, né? E eu não estou tendo condição de fazer pagando agora, não. É muito dinheiro. Não com tantas despesas. (UBS D, I1).

Aí, eu fiz os meus exames todos particulares, porque, pra fazer pela rede pública [silêncio, como se quisesse dizer que esperaria muito]... Eu passei uns dois anos assim: fazia e, quando ia fazer o outro, estava vencido aquele; e quando voltava no médico, já precisava fazer novamente [referindo-se à morosidade para realizar exames pelo SUS]. (UBS C, I1).

Pesquisa aponta a falta de comunicação entre os níveis de atenção como fragmentadora do cuidado e comprometedora da integralidade das ações ${ }^{54}$. O idoso é encaminhado a outros níveis de atenção quando a APS não 
oferece o serviço de que ele necessita, como, por exemplo, a realização de exames que demandam maior densidade tecnológica ${ }^{55}$. Desta forma, a demanda pela AE torna-se maior em relação ao serviço ofertado, gerando enormes filas de espera e ocasionando a fragmentação da linha de cuidado do idoso.

O usuário 'UBS C, I1' refere dificuldade, tanto para realizar o exame como para retornar ao médico. Isto demonstra que não há acesso adequado, e que a comunicação entre os pontos de atenção é falha, fazendo com que o idoso percorra longa trajetória, com custos sociais ${ }^{51}$. Além disto, aumentam-se os custos financeiros na $\mathrm{AE}$, já que o usuário necessita refazer os exames, por não ser atendido na consulta de retorno em tempo hábil.

\section{Considerações finais}

O percurso realizado pelos idosos ainda se distancia do previsto na legislação e evidencia barreiras de acesso traduzidas em dificuldades que impactam na resolutividade do cuidado. Evidenciou-se que, apesar de o modelo de atenção ainda ser fragmentado e estar distante da situação ideal, na qual estão presentes todos os atributos da APS, os usuários sentem-se satisfeitos com os cuidados recebidos, e identificam humanização e vínculos solidários no cuidado em saúde.

Entre as fragilidades, destacaram-se carências de recursos materiais e medicamentos, de infraestrutura e recursos humanos, dificuldade na acessibilidade aos serviços e falha na comunicação entre os níveis de atenção.

As UBS têm grande potencial, e este é percebido pelos usuários. Entretanto, a organização de seus processos de trabalho e sua articulação com os demais níveis de atenção à saúde precisam ser revistas e adequadas às especificidades desse ciclo de vida.

\section{Colaboradoras}

Sacco RCCS (0000-0001-6131-0852)* contribuiu substancialmente para concepção, planejamento, análise, interpretação dos dados e redação do artigo. Magalhães RG (0000-00017851-7227)* contribuiu para análise e interpretação dos dados, e redação do artigo. Assis MG (0000-0003-1636-555X)*, Guimarães SMF (0000-0002-2097-2355)* e Escalda PMF (0000-0003-0021-1193)* contribuíram igualmente para revisão crítica e aprovação final do conteúdo. 


\section{Referências}

1. Vello LS, Popim RC, Carazzai EM, et al. Saúde do Idoso: percepções relacionadas ao atendimento. Esc. Anna Nery. Rio de Janeiro. 2014; 18(2):330-335.

2. Closs VE, Schwanke CHA. A evolução do índice de envelhecimento no Brasil, nas suas regiões e unidades federativas no período de 1970 a 2010. Rev. Bras. Geriatr. Gerontol. 2012; 15(3);443-58.

3. Companhia de Desenvolvimento e Planejamento de Brasília. Pesquisa Distrital por Amostra de Domicílios. Ceilândia e Brazlândia. 2018. Brasília, DF: Secretaria de Estado de Planejamento e Orçamento do Distrito Federal; 2018.

4. Meireles VC, Matsuda LM, Coimbra JAH, et al. Características dos idosos em área de abrangência do Programa Saúde da Família na região noroeste do Paraná: contribuições para a gestão do cuidado em enfermagem. Saúde Soc. São Paulo. 2007; 16(1):69-80.

5. Schmidt MI, Ducan BB, Silva GA, et al. Doenças crônicas não transmissíveis no Brasil: cargas e desafios atuais. The Lancet. 2011; 9:61-74.

6. Gonçalves LHT, Alvarez AM, Sena ELS, et al. Perfil da família cuidadora de idosos doente/fragilizado do contexto sociocultural de Florianópolis, SC. Texto Contexto Enferm. 2006; 15(4):570-77.

7. Organização Mundial de Saúde. Relatório Mundial do Envelhecimento e Saúde. Genebra: Organização Mundial de Saúde; 2015.

8. Organización Panamericana de la Salud. Redes Integradas de Servicios de Salud: Conceptos, Opciones de Política y Hoja de Ruta para su Implementación en las Américas. Serie: La Renovación de la Atención Primaria de Salud en las Américas. Washington, D.C. $n^{\circ} 4.2010$

9. Brasil. Secretaria de Estado de Saúde do Distrito Federal. Portaria $\mathrm{n}^{\circ}$ 77, de 14 de fevereiro de 2017. Publicada no Diário Oficial do Distrito Federal no 33 , se- ções I, II e III de 15 de fevereiro de 2017. Estabelece a Política de Atenção Primária à Saúde do DF. 15 fev 2017. [acesso em 2020 jul 14]. Disponível em: http:// saude.df.gov.br/wp-conteudo/uploads/2018/04/ Portaria-SES_DF-n\%C2\%BA-77-2017-Esstabelece-a-Pol\%C3\%ADtica-de-Aten\%C3\%A7\%C3\%A3o-Prim\%C3\%Alria-\%C3\%A0-Sa\%C3\%BAde-do-Distrito-Federal.pdf.

10. Brasil. Ministério da Saúde. Secretaria de Atenção à Saúde. Saúde do Idoso. [acesso em 2019 set 1]. Disponível em: http:www.portal.saude.gov.br.

11. Lima-Costa MF, Matos DL, Camargos VP, et al. Tendências em dez anos das condições de saúde de idosos brasileiros: evidências da Pesquisa Nacional por Amostra de Domicílios (1998, 2003, 2008). Ciênc. Saúde Colet. 2011; 16(9):3689-3696.

12. Brasil. Ministério da Saúde. Política Nacional da Atenção Básica. 2017. [acesso em 2020 jul 14]. Disponível em: https://bvsms.saude.gov.br/bvs/saudelegis/ gm/2017/prt2436_22_09_2017.html.

13. Tesser CD, Neto PP, Campos GW. Acolhimento e desmedicalização social: um desafio para as equipes de saúde da família. Ciênc. Saúde Colet. 2010; 15(3):3515624.

14. Brasil. Ministério da Saúde. Portaria n ${ }^{\circ} 4279$, de 30 de dezembro de 2010. Estabelece diretrizes para a Rede de Atenção à Saúde. Diário Oficial da União. 30 Dez 2010. [acesso em 2020 jul 10]. Disponível em: https://bvsms.saude.gov.br/bvs/saudelegis/gm/2010/ prt4279_30_12_2010.html.

15. Gerhardt TE, Pinheiro R, Ruiz ENF, et al. Itinerários Terapêuticos e suas múltiplas dimensões desafios para a prática da integralidade e do cuidado como valor. In: Pinheiro R, Mattos RA. Razões Públicas para a integralidade em saúde: o cuidado como valor. 2 . ed. Rio de Janeiro: UERJ-ABRASCO; 2009. p. 279-300.

16. Cabral ALLV, Martinez-Hemaez A, Andrade EIG, et 
al. Itinerários terapêuticos: o estado da arte da produção científica no Brasil. Ciênc. Saúde Colet. 2011; 16(11):4422-4442.

17. Kessner DM, Kalk CE, Singer J. Assessing health quality - the case for tracers. N. Engl. J. Med. 1973; 288(4):189-94.

18. Feuerwerker LCM, Merhy EE. Como temos armado e efetivado nossos estudos, que fundamentalmente investigam políticas e práticas sociais de gestão e de saúde? In: Mattos RA, Baptista TWF, organizadores. Caminhos para análise das políticas de saúde. Porto Alegre: Rede UNIDA, 2015, p. 439-460.

19. Companhia de Desenvolvimento e Planejamento de Brasília. Pesquisa Distrital por Amostra de Domicílios (PDAD): Microdados Ceilândia e Brazlândia. Brasília, DF: CODEPLAN; 2018.

20. Oliveira MLC, Amâncio TG, organizadoras. Situações de saúde, vida e morte da população idosa residente no Distrito Federal. Curitiba: CRV; 2016.

21. Gondim SMG. Grupos focais como técnica de investigação qualitativa: desafios metodológicos. Paidéia. 2003; 12(24):149-161.

22. Bardin L. Análise de conteúdo. São Paulo: 70. ed.; 2016.

23. Fundação Instituto Brasileiro de Geografia e Estatística. Sistema de Referência Geocêntrico para as Américas (SIRGAS). Rio de Janeiro: IBGE; 2000.

24. Fernandes BL, Borgato MHA. Viuvez e a Saúde dos Idosos: uma Revisão Integrativa. Revista Kairós. Gerontologia. São Paulo. 2016; 19(3):187-204.

25. Aquino GA, Cruz DT, Silvério MS, et al. Fatores associados à adesão ao tratamento farmacológico em idosos que utilizam medicamento anti-hipertensivo. Rev. Bras. Geriatr. Gerontol. Rio de Janeiro. 2017; 20(1):116-127.

26. Merhy EE. A perda da dimensão cuidadora na produ- ção de saúde: uma discussão do modelo assistencial e da intervenção no seu modo de trabalhar a assistência. In: Campos CR, organizador. Sistema Único de Saúde em Belo Horizonte: reescrevendo o público. São Paulo: Xamã; 1998. p. 103-20.

27. Fonseca HLP. A reforma de saúde de Brasília, Brasil. Ciênc. Saúde Colet. 2019; 24(6):1981-1990.

28. Glote CRM, Gerhardt TE. Itinerários terapêuticos: integralidade no cuidado, avaliação e formação em saúde. As trajetórias assistenciais revelando à rede de atenção à saúde dos portadores de doenças cardiovasculares. In: Pinheiro R, Mattos RA. Razões Públicas para a integralidade em saúde: o cuidado como valor. 2. ed. Rio de Janeiro: UERJ-ABRASCO; 2016.

29. Raupp LM, Dhein G, Medeiros CRG, et al. Doenças crônicas e trajetórias assistenciais: avaliação do sistema de saúde de pequenos municípios. Physis. 2015; 25(2).

30. Batista SR, Vilarins GCM, Lima MG, et al. O Complexo Regulador em Saúde do Distrito Federal, Brasil, e o desafio da integração entre os níveis assistenciais. Ciênc. Saúde Colet. Distrito Federal. 2019; 24(6):27.

31. Brasil. Ministério da Saúde. Portaria ${ }^{\circ} 4.279$, de 30 de dezembro de 2010. Estabelece diretrizes para a organização da rede de atenção à saúde no âmbito do Sistema Único de Saúde (SUS). Diário Oficial da União. 30 Dez 2010. seção I, página 89.

32. Brasil. Ministério da Saúde. Portaria n ${ }^{\circ}$ 2048/GM, de 05 de novembro de 2002. Diário Oficial União. 2002 Nov 5. [acesso em 2020 jul 10]. Disponível em: https://bvsms.saude.gov.br/bvs/saudelegis/gm/2002/ prt2048_05_11_2002.html.

33. Alves MLF, Guedes HM, Martins JCA, et al. Rede de referência e contrarreferência para o atendimento de urgências em um município do interior de Minas Gerais - Brasil. Rev. Med. Minas Gerais. 2015; 25(4):469475 .

34. Cunha EM, Giovanella L. Longitudinalidade/conti- 
nuidade do cuidado: identificando dimensões e variáveis para a avaliação da Atenção Primária no contexto do sistema público de saúde brasileiro. Ciênc. Saúde Colet. 2011; 16(1):1029-1042.

35. Spedo SM, Pinto NRS, Tanaka OY. O difícil acesso a serviços de média complexidade do SUS: o caso da cidade de São Paulo, Brasil. Physis. Rio de Janeiro. 2010; 20(3):953-972.

36. Uchimura KY, Bosi MLM. Qualidade e subjetividade na avaliação de programas e serviços em saúde. Cad. Saúde Pública. Rio de Janeiro. 2002; 18(6):1561-1569.

37. Starfield B. Atenção primária: equilíbrio entre necessidades de saúde, serviços e tecnologia. Brasília, DF: Organização das Nações Unidas para a Educação, a Ciência e a Cultura; Ministério da Saúde; 2002.

38. Fagundes S. Apresentação. In: Ortiz JN, Bordignon MO, Gralha RS, et al. Acolhimento em Porto Alegre: um SUS de todos para todos. Porto Alegre: Prefeitura Municipal de Porto Alegre; 2004. p. 11-2.

39. Souza ECF, Vilar RLA, Rocha NSPD, et al. Acesso e acolhimento na atenção básica: uma análise da percepção dos usuários e profissionais de saúde. Cad. Saúde Pública, Rio de Janeiro. 2008; 24(1):100-110.

40. Uchoa AC, Souza EL, Spinelli AFS, et al. Avaliação da satisfação do usuário do Programa de Saúde da Família na zona rural de dois pequenos municípios do Rio Grande do Norte. Physis, Rio de Janeiro. 2011; 21(3):1061-1076.

41. França AJ, Andrade FA, Araújo TM, et al. Relação intraequipe e produção de vínculos em uma Unidade de Saúde da Família. EFDeportes. 2014; 18(190).

42. Donabedian A. An introduction to quality assurance in health care. New York: Oxford University, 2003.

43. Franco TB, Bueno WS, Merhy EE. O acolhimento e os processos de trabalho em saúde: o caso de Betim, Minas Gerais, Brasil. Cad. Saúde Pública. 1999; 15(2):345-53.
44. Silva KL, Sena RR, Seixas CT, et al. Desafios da política, da gestão e da assistência para a promoção da saúde no cotidiano dos serviços. REME - Rev. Min. Enferm. 2012; 16(2):178-187.

45. Conill EM. Políticas de atenção primária e reformas sanitárias: discutindo a avaliação a partir da análise do Programa saúde da Família em Florianópolis, Santa Catarina, Brasil, 1994-2000. Cad. Saúde Pública. 2002; 18(1):191-202.

46. Brasil. Lei $\mathrm{n}^{0} 10.741$, de 01 de outubro de 2003. Dispõe sobre o Estatuto do Idoso e dá outras providências. Diário Oficial da União. 1 Out 2003 [acesso em 2020 jul 10]. Disponível em: http://www.planalto. gov.br/ccivil_03/leis/2003/110.741.htm.

47. Martins MS, Massarollo MCKB. Mudanças na assistência ao idoso após promulgação do estatuto do idoso segundo profissionais de um hospital geriátrico. Rev. Esc. Enferm. USP 2008; 42(1):26-33.

48. Parente AS, Mesquita FOS, Oliveira MR. Satisfação dos idosos atendidos pela estratégia de saúde da família em um município do interior de Pernambuco. Rev. Adm. Saúde. 2017; 17(68).

49. Viacava F, Oliveira RAD, Carvalho CC, et al. SUS: oferta, acesso e utilização de serviços de saúde nos últimos 30 anos. Ciênc. Saúde Colet. Rio de Janeiro. 2018; 23(6):1751-1762.

50. Brasil. Ministério da Saúde. Política Nacional de Medicamentos. Aprovada pela Portaria no 3.916, de 01 de outubro de 1998. Brasília: 1998. [acesso em 2020 jul 14]. Disponível em: https://bvsms.saude.gov.br/ bvs/saudelegis/gm/1998/prt3916_30_10_1998.html.

51. Paim JS. Sistema Único de Saúde (SUS) aos 30 anos. Ciênc. Saúde Colet. 2018; 23(6):1723-1728.

52. Arakawa AM, Simone ALH, Magali LC, et al. Percepção dos usuários do sus: expectativa e satisfação do atendimento na Estratégia de Saúde da Família. Rev. CEFAC. 2012; 14(6):1108-1114. 
53. Arruda C, Lopes SGR, Koerich MHAL, et al. Redes de atenção à saúde sob a luz da teoria da complexidade. Esc. Anna Nery. 2015; 19(1):169-173.

54. Gouveia GC, Souza WV, Luna CF, et al. Satisfação dos usuários do sistema de saúde brasileiro: fatores associados e diferenças regionais. Rev. Bras. Epidemiol. 2009; 12(3):281-96.
55. Santos CTB, Andrade OLM, Silva MJ, et al. Percurso do idoso em redes de atenção à saúde: um elo a ser construído. Physis. 2016; 26(1):45-62.

Recebido em 19/12/2019

Aprovado em 08/06/2020

Conflito de interesses: inexistente

Suporte financeiro: Fundação de Apoio à Pesquisa do Distrito

Federal (FAP/DF). Coordenação de Aperfeiçoamento de Pessoal de Nível Superior (Capes), Código de Financiamento 001 